\title{
Rotational Speed Direction Detection Based on the Output Duty Cycle of a Differential Hall Effect Gear Tooth Sensor
}

\author{
Cheng Liu¹, Jianhai Qiu², Ji-Gou Liu' \\ ${ }^{1}$ ChenYang Technologies GmbH \& Co. KG, Markt Schwabener Str. 8, 85464 Finsing, Germany \\ cheng.liu@chenyang-ism.com \\ 2 University of Shanghai for Science and Technology, Jungong Rd. 516, 200093 Shanghai, China
}

\begin{abstract}
:
In this paper, a new method is proposed to use the duty cycle of the output signal from a differential Hall Effect gear tooth sensor for determining the rotational direction. The magnetic polarization of the detected differential magnetic field density is changed by changing the rotational direction of the target wheel. Therefore a variation in duty cycle of the electrical output signal is visible when the geometric duty cycle of the target wheel is designed about $70 \%$. This method works with special target gears which are designed and optimized by magnetic field simulation and experiments. The presented method is applied to the differential Hall Effect gear tooth sensor CYGTS101DC-S, which has a large sensing gap of $3.5 \mathrm{~mm}$, a relatively stable duty cycle of $61.24 \%$ in clockwise direction and of $38.76 \%$ in counter-clockwise direction under using the designed target wheel.
\end{abstract}

Key words: Hall Effect Gear Tooth Sensors, Duty Cycle, Rotational Speed, Rotational Direction, Magnetic Field Simulation

\section{Introduction}

Rotational speed measurement is an important operation for applications in industry and automation. Especially in electrical drive systems, rotational speed sensors are essential for a proper functionality of the whole machine [1].

There exist various kinds of rotational speed sensors based on different physical principles. Most of them use the pulses counting method to measure speed with a target wheel [3].

The rotational direction can be determined only by using two built-in detectors [2, 3, 4]. Both detectors are positioned in a distance of onefourth gear pitch in order to generate two series of pulses, the phase shift of which is $90^{\circ}$ (see Fig. 1). The direction of rotation can be detected with a flip-flop circuit. However, an additional detector needs higher production costs and larger size of sensor case etc.

In this paper, a new method for determining the rotational direction is introduced by using the duty cycle of the sensor output signal. According to the theoretical analysis and experimental results, the duty cycle of the output signal of a differential sensor changes with the rotational direction of the target wheel.

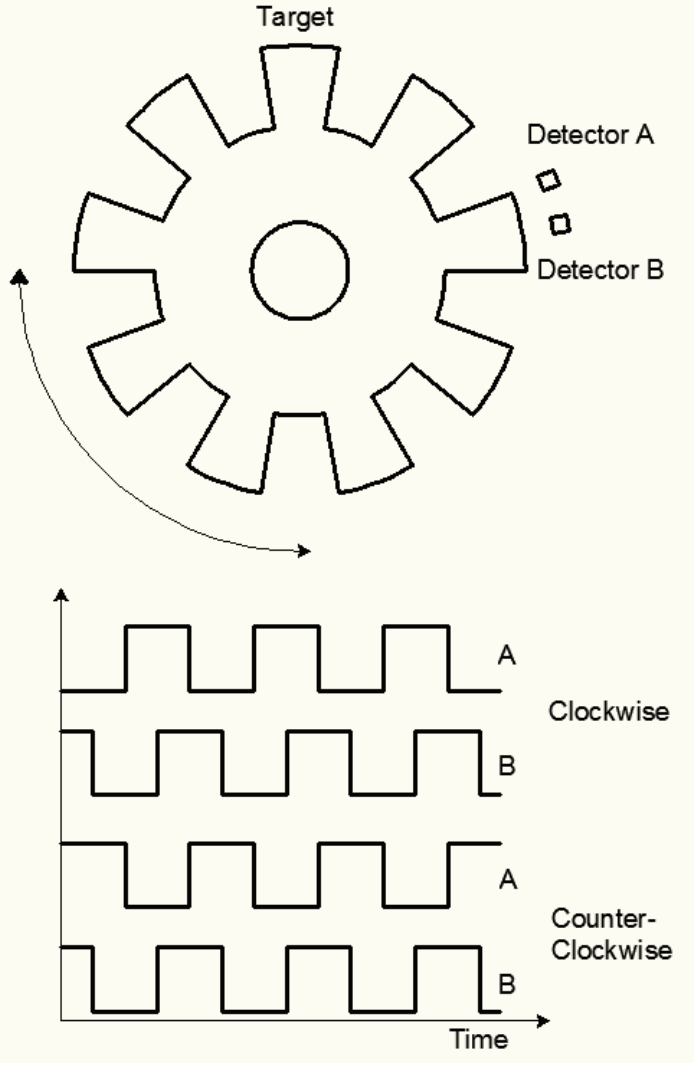

Fig. 1. Rotational direction detection by using two detectors 
Therefore the rotational direction of the target wheel can be determined by detecting the polarity change of differential magnetic field density between the two Hall probes of the detector (see Fig. 2). In this way the sensor structure and signal processing are simplified.

\section{Speed Direction Determination by Duty Cycle}

The duty cycle of the output signal from a differential Hall Effect gear tooth sensor can be used for the determination of rotational direction, when the sensor is coupled with an optimized target gear. As illustrated in Fig. 2, a differential gear tooth speed measuring system consists of a target gear and a differential Hall Effect gear tooth detector, in which a permanent magnet and two Hall probes are integrated.

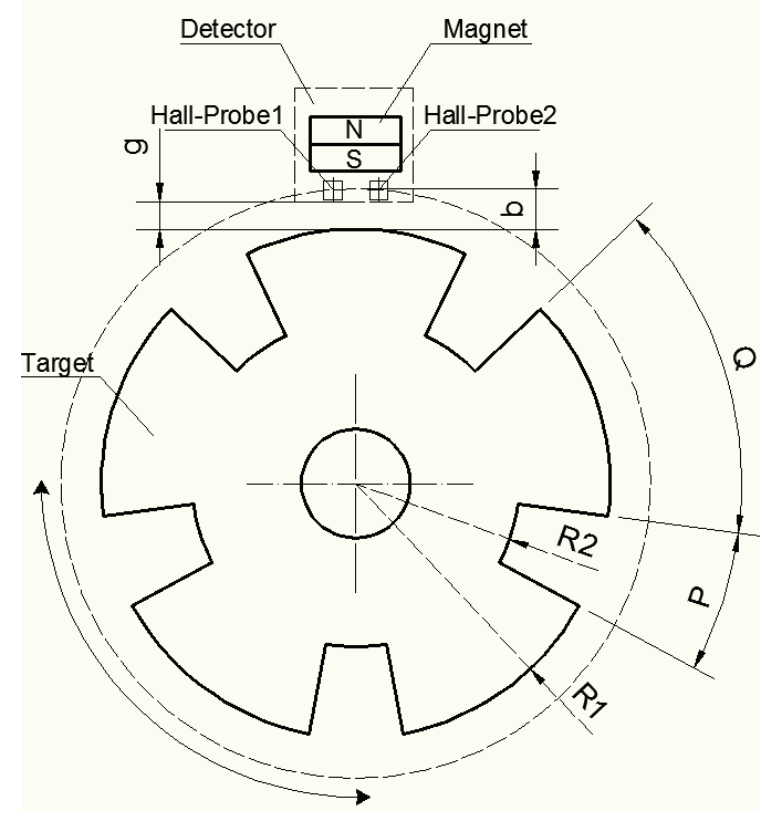

Fig. 2. Differential gear tooth speed measuring system with two Hall probes

The distance between the Hall probes and the target addendum is $b$, while the gap $g$ is the distance between detector and target addendum. For appropriate design of the target wheel, the parameters $Q, P, R 1$ and $R 2$ are essential, which stand for the tooth width, the slot width, the radius of the target addendum and dedendum.

Fig. 3 shows the differential magnetic field density between both probes, which is given as:

$$
\Delta B=B 1-B 2
$$

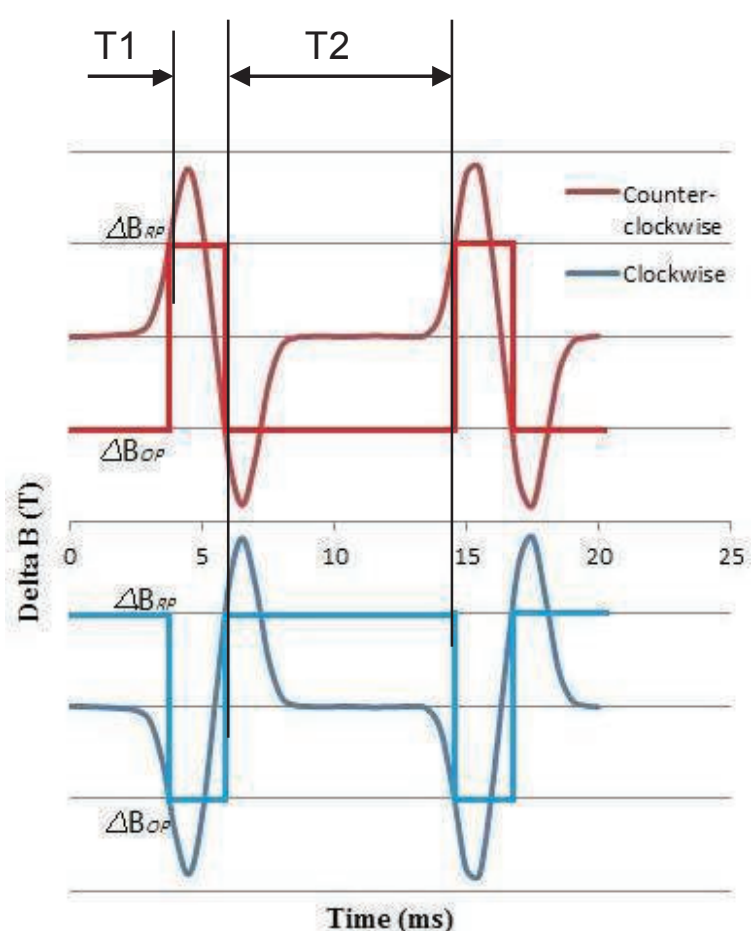

Fig. 3. Differential magnetic field density and output impulses of Hall detector in both rotational directions

where $B 1$ and $B 2$ are the measured magnetic field densities of Hall probes 1 and 2. $\Delta B_{O P}$ presents for the differential magnetic field density at operational point and $\Delta B_{R P}$ at the release point.

As shown in Fig. 3, the differential magnetic field density $\Delta B_{C W}$ in clockwise rotational direction is reversed to that $\Delta B_{C C W}$ in counterclockwise direction, i.e.:

$$
\Delta B_{C W}=-\Delta B_{C C W}
$$

Therefore the direction change can be detected by the polarity change of differential magnetic field density.

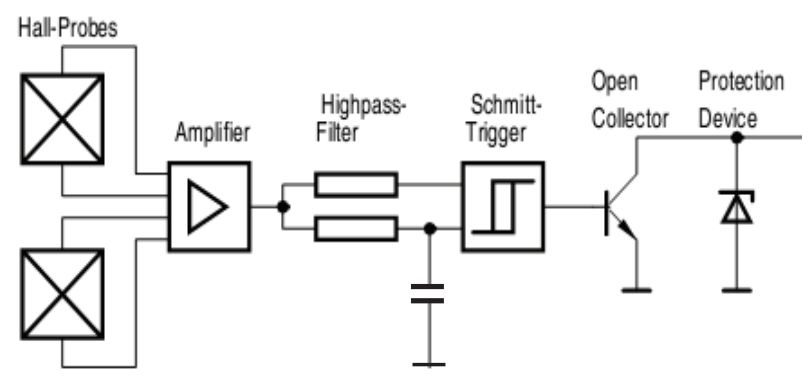

Fig. 4. Block diagram of the used detector 
After converting the differential magnetic field density to impulses by a built-in Schmitt-Trigger (see Fig.4), the output signal of the detector will reverses the high to low level (or vice versa) when the rotational direction is changed. The output impulses of clockwise and counterclockwise are complementary. It can be derived that the sum of the duty cycles in the clockwise and counter-clockwise directions is equal to $100 \%$ :

$$
D C_{C W}+D C_{C C W}=100 \%
$$

with $D C_{C W}$ and $D C_{C C W}$ as the duty cycle in the clockwise direction and in the counter-clockwise direction, respectively.

If the duty cycle of the output pulses for the counter-clockwise rotation is determined by

$$
D C_{C C W}=\frac{T 1}{T 1+T 2} \times 100 \%
$$

The duty cycle of the output pulses for the clockwise rotation can be then calculated by

$$
D C_{C W}=100 \%-D C_{C C W}=\frac{T 2}{T 1+T 2} \times 100 \%
$$

This property of the duty cycle can be used for detect the rotational direction. By using an optimized geometric duty cycle of the target wheel:

$$
D C_{g}=\frac{Q}{Q+P} \times 100 \%
$$

one can obtain different duty cycle $D C_{C W}$ and $D C_{C C W}$ for detecting the rotational direction.

For a better detection of the rotational direction, $D C_{C W}$ and $D C_{C C W}$ should become significant different. This can be realized by optimizing the geometric parameters of the target wheel like the tooth width $Q$, the slot width $P$ and the number of teeth $N$. Therefore, simulation and experiments should be done in order to optimize the target wheel for rotational measurement with direction detection.

\section{Experimental Results}

Experiments by using different target gears are made in order to optimize the tooth width $Q$ and slot width $P$. An experimental setup shown in Fig. 5, which consists of an oscilloscope (1) and a motor test stand (2), was used for the experiments. On the motor test stand (see Fig. 6 ), a target wheel (4) is mounted on the shaft of the motor (3). A speed sensor under test (5), for instance a Hall Effect gear tooth sensor coupled with the target wheel, is used to measure the rotational speed of the motor. The sensor output signal is sampled with the oscilloscope.

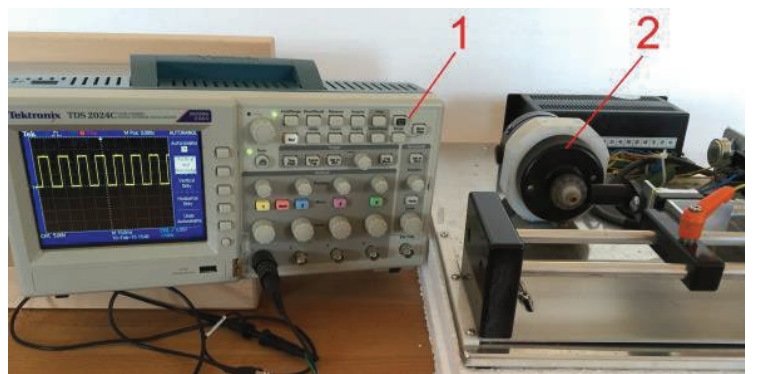

Fig. 5. Experimental setup

Under using this experimental setup, target gears with different $Q / P$ ratio are tested. Fig. 7 shows the forms of three different target wheels under test. By knowing the tooth width $Q$ and slot width $P$ of each gear, the geometric duty cycle $D C_{g}$ can be calculated according to (6)

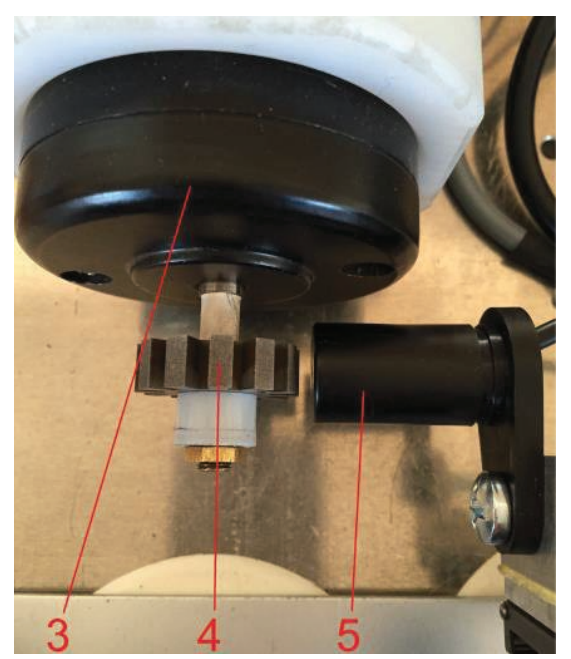

Fig. 6. Motor test stand

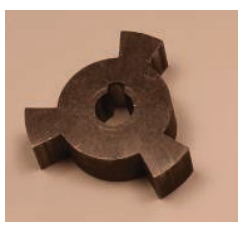

Target 1

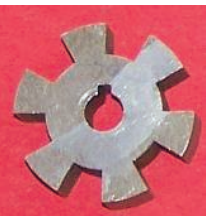

Target 2

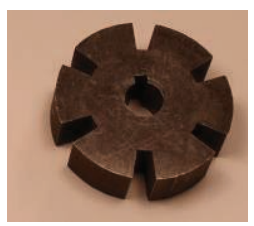

Target 3
Fig. 7. Target wheels for experiment 
Tab. 1: Geometric duty cycle and Q/P ratio of each target wheel

\begin{tabular}{|c|c|c|c|}
\hline Target & 1 & 2 & 3 \\
\hline$Q / P$ & $10.56 / 18.76$ & $7.33 / 7.33$ & $10.26 / 4.4$ \\
\hline$D C_{g}$ & $36.0 \%$ & $50.0 \%$ & $70.0 \%$ \\
\hline
\end{tabular}

The $Q / P$ ratio and the geometric duty cycle $D C_{g}$ of each target gear are listed in Tab. 1.

The duty cycle of these three target gears can be determined by experiments. Fig. 8 shows the relative deviation between the measured duty cycles and the geometric duty cycles. Apparently, the measured duty cycle is more approximately to the geometric duty cycle if the $Q / P$ is larger. Therefore, a target wheel with a larger $Q / P$ ratio is preferred to use in order to get a better accuracy and estimation of the duty cycle of the output signal according to the geometric duty cycle.

However, a target wheel with excessively large $Q / P$ ratio, such as $8 / 2$, causes a decreasing differential magnetic field density. This results in a smaller sensing gap for the sensor.

Due to the manufacturing errors of the sensor, for example, the positional error of the Hall probes, the duty cycle of the output signal may deviate from its true value. Therefore a $Q / P$ ratio of 6/4, which has a geometric duty cycle of $60 \%$, is not a good choice. The duty cycles in both rotation directions may be about $50 \%$, so that the direction couldn't be easily determined correctly.

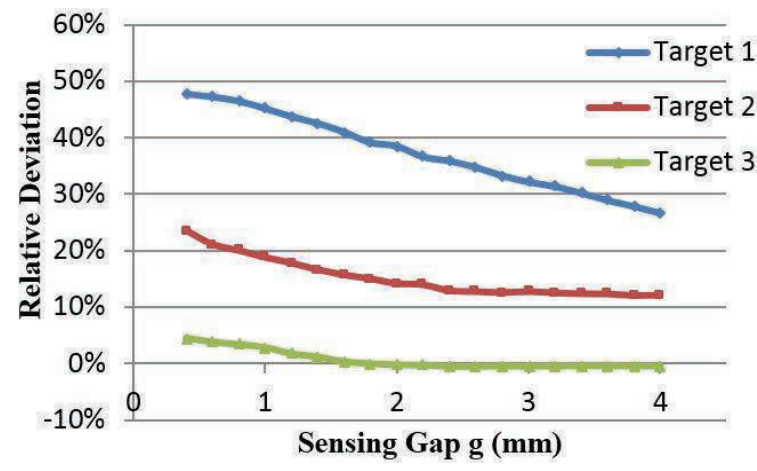

Fig. 8. Relative Deviation between geometric duty cycle and measured duty cycle of three targets gears

Based on the analysis above, a $Q / P$ ratio of $7 / 3$ is recommended as the best $Q / P$ ratio, thanks to its good performances for estimating the duty cycle accurately and for getting a larger sensing air gap in its applications.

\section{Simulation Results}

Magnetic Field Simulation (MFS) has been well established for the design of Hall Effect gear tooth sensors [7]. The MFS is realized with the Ansoft Maxwell software. Ansoft Maxwell is a powerful, FEM-based and commercial software for electromagnetic field simulation used for the design and analysis of 2D/3D structures. By application of the Maxwell magnetic field theory, this software can be used for calculating static and dynamic electromagnetic fields.

\subsection{Simulation Model}

For the purpose of getting reliable simulation results, the simulation model has to be verified by real measurements. Fig. 9 shows the simulation model of a differential gear tooth speed measuring system under using Ansoft Maxwell. The parameters for the simulation components are set in Tab. 2.

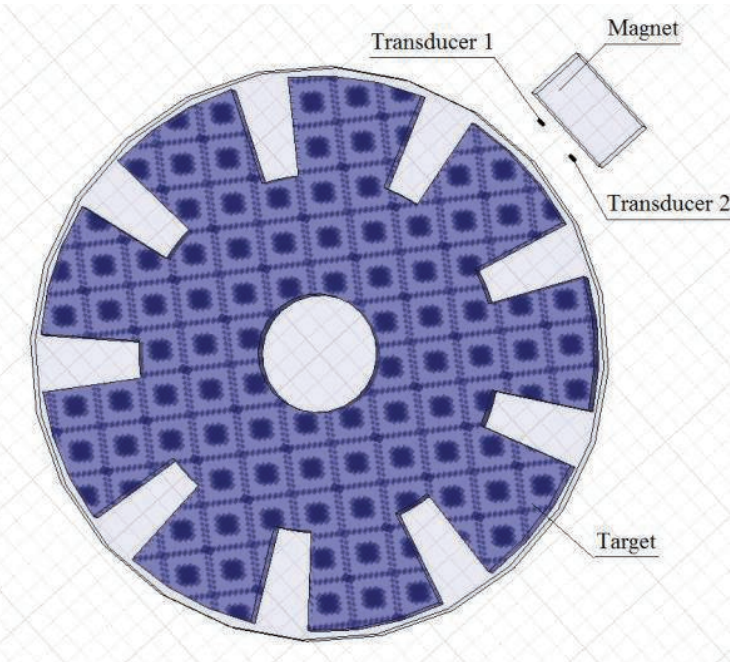

Fig. 9. Magnetic field simulation model of differential gear tooth measuring system under using Ansoft Maxwell

For instance, the signal duty cycle of a 5 teeth target gear is analyzed with this simulation model. Based on the differential Hall Effect gear tooth sensor CYGTS101DC-S, whose release point and operate point are at $\pm 0.75 \mathrm{mT}$ [9], the duty cycles in clockwise and counter-clockwise direction are obtained. The measurements are done on the motor test stand. Results show that the deviations between the duty cycles $\left(D C_{C W}\right.$ and $\left.D C_{C C W}\right)$ of the simulation and measurements are less than $4 \%$ with a sensing gap $g$ from $0.5 \mathrm{~mm}$ to $4 \mathrm{~mm}$, see Fig. 10 . This simulation model can be used for the estimation of the duty cycles. 
Tab. 2: Parameters of the simulation components for the simulation model

\begin{tabular}{|c|c|c|c|c|}
\hline Component & Material & $\begin{array}{c}\text { Max } \\
\text { Number of } \\
\text { Mesh } \\
\text { Elements }\end{array}$ & \multicolumn{2}{|c|}{$\begin{array}{c}\text { Size } \\
(\mathrm{mm})\end{array}$} \\
\hline Magnet & SmCo17 & 80000 & \multicolumn{2}{|c|}{$8 \times 5 \times 3$} \\
\hline Target & Steel_1010 & 100000 & $\begin{array}{c}\text { R1 } \\
=14\end{array}$ & $\begin{array}{r}\text { R2 } \\
=9\end{array}$ \\
\hline Hall Probes & Vacuum & 80000 & $0.1 \times 0.1$ \\
\hline Region & Vacuum & 150000 & $4000 \%$ \\
\hline
\end{tabular}

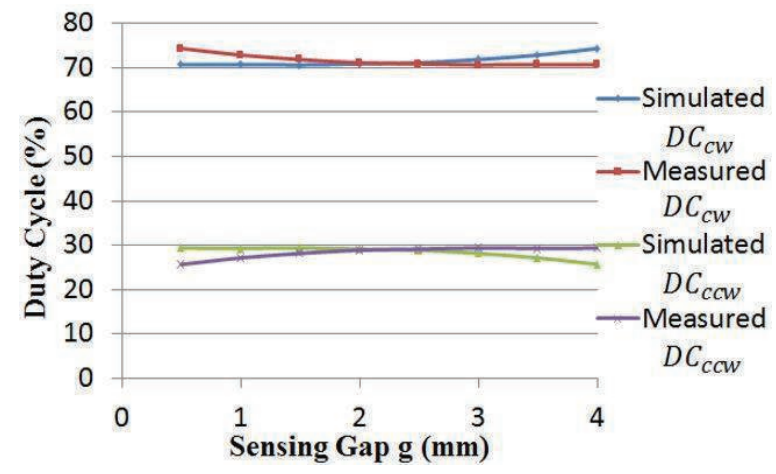

Fig. 10. Duty cycles in clockwise and counterclockwise direction from simulation and measurements

\subsection{Optimizing the Number of Teeth}

Another important parameter of the target gear is the number of teeth $N$. A higher number $N$ means more pulses per revolution and therefore a higher resolution of the sensor. However, $N$ is limited by the fact that the sensing gap decreases with increasing teeth number. The reason is that a smaller slot width $P$ leads to a smaller differential magnetic field density [7]. Additionally, a target gear with more teeth may be inappropriate for rotational direction determination. Its duty cycles in clockwise and counter-clockwise directions are about $50 \%$. Due to manufacturing and installation errors and vibrations, the duty cycles may be unstable. This causes incorrect rotational direction determination. For achieving the best performance in sensing gap, sensor resolution and direction determination, the number of teeth $N$ has to be optimized.

The teeth number $N$ can be optimized by the MFS. A target gear model with Q/P ratio of $7 / 3$ is used with different number of teeth $(5,6,8$, 9). Fig. 11 shows the simulation results of the duty cycles in clockwise direction. The results illustrates that the duty cycle remains relatively stable with increasing sensing air gap $g$. The target gear with 5 teeth can even reach a sensing gap of $4 \mathrm{~mm}$. The maximum sensing air gap for the other target gears is $3.5 \mathrm{~mm}$. So the sensing air gap $g$ decreases with increasing the number of teeth $N$.

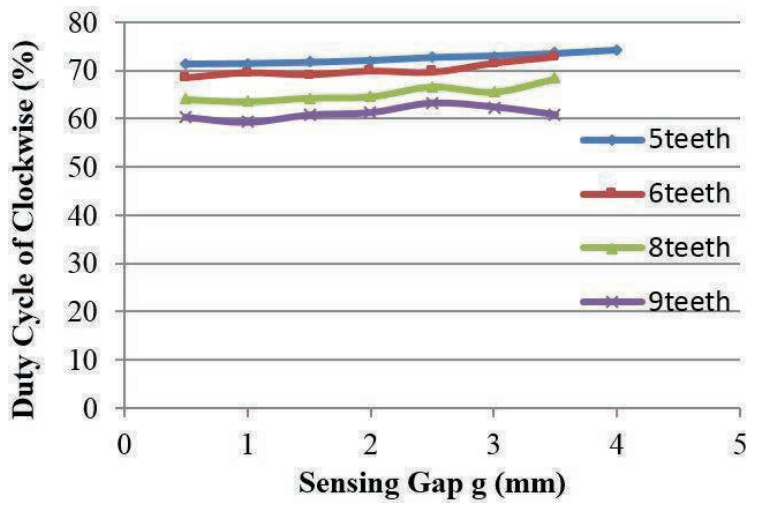

Fig. 11. Duty cycles in clockwise direction with different number of teeth

Nevertheless, a target gear with nine teeth provides the best compromise between large sensing air gap and high resolution of the rotational speed sensor. It can be used with a sensing gap of $3.5 \mathrm{~mm}$. Moreover, it is also suitable for rotational direction determination, with an average duty cycle of $61.24 \%$ in clockwise direction and of $38.76 \%$ in counterclockwise direction.

\section{Rotational Speed Measurement with Direction Detection}

For detecting the rotational direction of the target wheel, the duty cycle of the output signal must be determined in the following way. A negation should be connected to the output of the sensor, in order get an additional complementary signal. An additional pulse signal with a high frequency is used for the interpolation, see Fig. 12. The impulse quantity is presented with $\mathrm{N} 1$ and $\mathrm{N} 2$.

The target wheel rotates in the counterclockwise direction if $\mathrm{N} 1<\mathrm{N} 2$. Otherwise the target wheel rotates in the clockwise direction $(\mathrm{N} 1>\mathrm{N} 2)$. The rotational speed can be determined by 


$$
\omega=\frac{60 N}{m T} \quad(\mathrm{rpm})
$$

with $m$ as the number of teeth, $N$ as the quantity of pulses and $T$ as measuring time, under using the pulse signal. In order to guarantee the detection of the rotational direction, the condition $\mathrm{Q}>2 \mathrm{P}$ should be fulfilled.

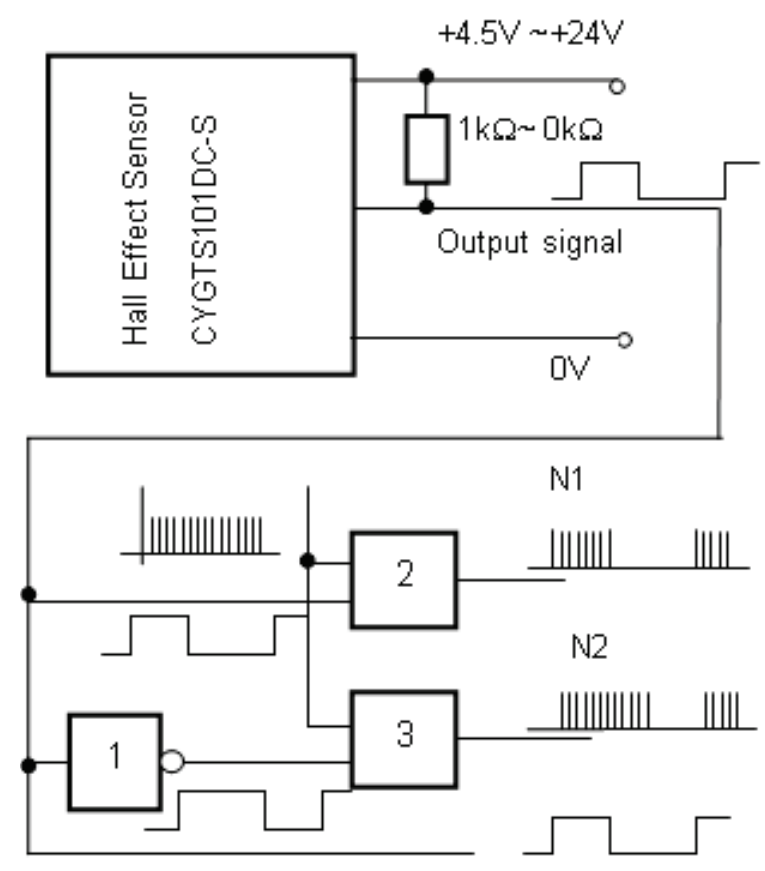

Fig. 12. Interpolation of the Sensor Output Signal with a High Frequency Pulse Signal for Rotational Direction Determination

\section{Conclusions}

In this paper, a new method for determining the rotational direction by using the duty cycle of the sensor output signal has been proposed. From the results one can draw the following conclusions:

- The output signal of a differential Hall Effect gear tooth sensor in the clockwise direction is the complementary signal in the counterclockwise direction due to the reverse of differential magnetic field. Therefore the duty cycle can be used for determining the rotational direction of drive systems.

- The tooth width $Q$, the slot width $P$ and the number of teeth $N$ of a target gear have to been optimized to achieve a reliable rotational direction determination.

- From the experimental results, a target gear with a geometric duty cycle of $70 \%$ is preferred, if it is coupled with the sensor CYGTS101DC-S. It provides a significant difference between the duty cycles in both directions.

- Magnetic Field Simulation (MFS) can be used for the optimization of a target gear. The deviation between the estimated duty cycles and the measured duty cycles is less than $4 \%$.

- From the simulation results, a target gear with nine teeth is the best choice in order to have a large sensing air gap and a high sensor resolution.

- Consequently, an optimized target gear for CYGTS101DC-S has to be designed with nine teeth for an outer diameter of $28 \mathrm{~mm}$ and a geometric duty cycle of $70 \%$. The sensing air gap reaches $3.5 \mathrm{~mm}$, whereas the average duty cycle is $61.24 \%$ in the clockwise direction and $38.76 \%$ in the counter-clockwise direction.

\section{References}

[1] D. Schröder: „Elektrische Antriebe - Regelung von Antriebssystemen", 2nd Edition, SpringerVerlag, Berlin, Heidelberg, New York, 2001.

[2] E. Ramsden: "Hall Effect Sensors - Theory and Application", Elsevier Inc., Amsterdam, London, New York etc., 2006.

[3] J.-G. Liu, Z. Zheng: "Mathematical Models of Gear Tooth Speed Sensors with Dual Outputs", Joint International IMEKO TC1+TC7+TC13 Symposium, pp. 82-86, Jena, Germany, 2011.

[4] E. Schrüfer: „Elektrische Messtechnik - Messung elektrischer und nichtelektrischer Größen“, 6th Edition, Carl Hanser Verlag, Munich, Germany, 1995.

[5] S. A. Macintyre: "Magnetic Field Measurement", CRC Press LLC, 1999.

[6] J. Lu, J.-G. Liu: "Parameter Optimization of Hall Effect Gear Tooth Speed Sensors", PCIM EUROPE International Exhibition and Conference for Power Electronics, Intelligent Motion, Power Quality, pp.1650-1657, Nuremberg, Germany, May 8-10, 2012.

[7] C. Liu, J. Lu, J.-G. Liu: "Design of Hall Effect Gear Tooth Speed Sensors by Using Magnetic Field Simulation", AMA Conferences 2013 Sensor 2013, 16th International Conference on Sensors and Measurement Technology, pp.675679, Nuremberg, Germany, May 14-16, 2013. DOI 10.5162/Sensor 2013/P1.4

[8] R. S. Popovic, "Hall Effect Devices", 2nd Edition, Institute of Physics Publishing, Bristol, 2004.

[9] ChenYang Technologies $\mathrm{GmbH}$ \& Co. KG: "Hall Effect Differential Gear Tooth Sensors CYGTS101DC-S", data sheet.

(http://www.hallsensors.de/CYGTS101DC-S.pdf) 